\title{
Correspondence
}

\author{
Editors: Andrew C. Smith and Grog Wilkinson
}

\section{The Continuum of Psychosis and the Gene}

SIR: Crow (Journal, October 1986, 149, 419-429) appears to fall into the trap, common to most discussions of unitary psychosis, of regarding psychosis or psychoses as disease(s) rather than syndrome(s). As a result, he is led to postulate the existence of a peculiarly unstable gene or limited set of genes whose abnormalities express themselves as psychosis of varying symptomatology.

That schizophrenia is a syndrome has been recognised ever since Eugen Bleuler wrote about "the schizophrenias", and the evidence that they have multiple aetiologies is now fairly compelling. Of particular relevance to Crow's argument is the finding that process schizophrenias may have a rather low heritability relative to some other types. Severe depression, also, seems to have multiple causes and it is probable that mania has more than one cause (it appears that, in a small number of families, its inheritance is X-linked while in most families it is not; some patients may have abnormal vanadium metabolism; some patients respond well to lithium while others do not, etc.). In situations like this, the observation of continua between syndromes is to be expected and has no great aetiological significance. After all, high dose steroids can produce depression in one patient, mania in another and paranoid schizophrenia in a third. It would be most interesting to know the reason for these idiosyncrasies, but they would hardly justify postulating the existence of a special 'steroid psychosis' gene with unique properties.

It would not be difficult to construct an argument similar to Crow's, centering around bellyache. There is considerable overlap in the symptoms and other characteristics of people suffering abdominal pain, and it may well be the case that children of women with gallstones are prone to develop diverticulitis (as a result of common nutritional habits). Equally, there are probably seasonality effects in bellyache since it is likely to be more common at Christmas time and over the summer holiday period. Perhaps, therefore, there is a bellyache gene similar to the proposed psychosis gene. There are no doubt genes controlling cerebral organisation and dominance effects which may one day be of great interest to psychiatrists especially, I suspect, those studying personality disorder, but existing knowledge is insufficient to enable one to say anything of heuristic value about them.

\section{Royal South Hants Hospital Southampton SO9 4PE}

C. M. H. NunN

SIR: Crow is to be congratulated on his imaginative theory of psychosis (Journal, October 1986, 149, 419-429). One thing puzzles me about his use of the season of birth data to support a viral explanation. His Figure 2 certainly shows, consistent with the hypothesis, that there is an excess of births in patients with psychotic illnesses during the winter months. However, it also shows an equally large reduction during the summer months, a phenomenon that seems to be difficult to reconcile with the presumed pathogenic effects of a virus.

There are several equally convincing explanations of the season of birth effect, one being that it reflects the cycles of sexual activity among the parents of future psychotic patients. Might this not be a more credible and parsimonious explanation of the data, given that there is no other evidence for the viral theory?

\section{Magdalen College}

Gordon Claridge Oxford OX14AU

\section{Chronic Schizophrenia and Long-term Hospitalisation}

SiR: It was good of Abrahamson (Journal, September $1986,149,382)$ to notice Institutionalism and Schizophrenia (Wing \& Brown, 1970), but I wonder if I could persuade him to re-read it? He will find that we did not describe one pattern of relationship between negative aspects of behaviour in schizophrenia and length of stay in hospital. There were three, a different pattern for each of our hospitals. More important, our conclusions about the influence of social environment were not derived from these results. On the contrary, we stated (p. 12) that there was overwhelming evidence against the "startling assumption that length of stay is in any way significant as a measure of severity of illness".

What we did find was that when age and length of stay were standardised, patients in the hospital with 